\title{
A Taxonomy for Cross-domain Fire Hazards in Building Design
}

\author{
Kalluri Mallikarjuna, Balaji; Kivac, Anja; Rosenqvist, Hannah
}

Published in:

30th European Safety and Reliability Conference and 15th Probabilistic Safety Assessment and Management Conference

Publication date:

2020

Document Version

Peer reviewed version

Link back to DTU Orbit

Citation (APA):

Kalluri Mallikarjuna, B., Kivac, A., \& Rosenqvist, H. (2020). A Taxonomy for Cross-domain Fire Hazards in Building Design. In P. Baraldi, F. Di Maio, \& E. Zio (Eds.), 30th European Safety and Reliability Conference and 15th Probabilistic Safety Assessment and Management Conference (pp. 3959-3966). Research Publishing Services.

\section{General rights}

Copyright and moral rights for the publications made accessible in the public portal are retained by the authors and/or other copyright owners and it is a condition of accessing publications that users recognise and abide by the legal requirements associated with these rights.

- Users may download and print one copy of any publication from the public portal for the purpose of private study or research.

- You may not further distribute the material or use it for any profit-making activity or commercial gain

- You may freely distribute the URL identifying the publication in the public portal 


\title{
A Taxonomy for Cross-domain Fire Hazards in Building Design
}

\author{
Balaji Kalluri \\ The Danish Institute of Fire and Security Technology, Hvidovre, Denmark. E-mail: bak@brandogsikring.dk \\ Technical University of Denmark, Lyngby, Denmark.E-mail: bakal@dtu.dk \\ Anja Kivac \\ The Danish Institute of Fire and Security Technology, Hvidovre, Denmark. E-mail: ank@brandogsikring.dk \\ Hannah Rosenqvist \\ The Danish Institute of Fire and Security Technology, Hvidovre, Denmark. E-mail: hro@ brandogsikring.dk
}

This study looks at the future of building designs, particularly Internet of Things (IoT) integrated systems in built environment. The value of such smart environments are clear, whereas the risk introduced by cross-domain fire safety hazards is ambiguous. The study elucidates several potential hazards that could compromise fire protection due to combination of cyber, social and physical means in smart homes. A taxonomy for cross-domain fire safety attack vectors and their impact is developed through scenario-based analysis. Furthermore, the taxonomy help identify seven means of attack, spread across three cross-domains that could potentially introduce four different types of fire-safety hazards in future smart buildings.

Keywords: cross-domain, fire-safety, hazards, smart buildings, taxonomy, IoT

\section{Introduction}

Contemporary building designs can be viewed as a System-of-Systems with higher degree of integration and interaction. Several efforts including but not limited to smart grids (Kolokotsa 2016), smart cities (Silva et al. 2018), low-carbon cities (Kennedy et al., 2014), net-zero energy buildings (Kylili and Fokaides, 2015) favour this paradigm shift in building design primarily to facilitate sustainable urban (re)development. Fig. 1 shows such a new paradigm of buildings as Systems-ofSystems. It presents how various design characteristics in contemporary buildings are interrelated. For instance, Building-integrated Photovoltaics (BIPV) together with on-site Energy Storage Systems (ESS) and Smart Energy Meters (EM) enable buildings an active participant in smart grids and cities. However, to make buildings smarter, various systems and services such as Electrical Systems (ES), Mechanical Systems (MS), Security Systems (SS), Active Fire Protection (AFP) systems, and other Miscellaneous Electrical Loads (MEL) within a building need to be coordinated and automated intelligently. This requires a Building Management System (BMS) as the backbone for integration of various subsystems and related Sensor and Actuators (S\&A) using Information Communication Technology (ICT) to gather all the information to/from the Building Automation System (BAS). BAS implements intelligent algorithms to learn, predict and make smart decisions cooperatively and in real-time. Of course, building designs also need to meet physical structural requirements which are collectively represented as Structural and Passive Design (SPD). This include subsystems such as facades, windows, floors, ceilings, etc. Advancements in new materials influence building designs as well. Smart Material (SM) properties such as phase-change, selfhealing and carbon sequestering find applications in cool paints, concrete mixture, glazing facades and so on. Furthermore, integration of Vegetation (VEG) in the form of green vertical walls and roof gardens aid to improve the social and psychological well-being of occupants, particularly in highly urbanized built-environment. Besides, this passive design feature also has proved effective in regulating heat loads internally within buildings.

There are several reasons favouring integrated building design practice in recent years, particularly with functional integration through information exchange between subsystems within the building (Wang et al. 2018). Some of the benefits are improved energy efficiency, occupant's comfort, well-being, facilities management, fire protection and evacuation as shown in Fig. 1. For instance, the implementation of sustainable/green building rating systems such as LEED (Council. 2001) and DK-DGNB ${ }^{a}$ for an entire building, energy efficiency labelling schemes such as Ener-

\footnotetext{
$\overline{{ }^{a} \text { https://www.dk-gbc.dk/ }}$
}

Proceedings of the 30th European Safety and Reliability Conference and the 15th Probabilistic Safety Assessment and Management Conference. Edited by Piero Baraldi, Francesco Di Maio and Enrico Zio Copyright (C) 2020 by ESREL 2020 PSAM 15 Organizers. Published by Research Publishing, Singapore ISBN: 981-973-0000-00-0 :: doi: 10.3850/981-973-0000-00-0_ 


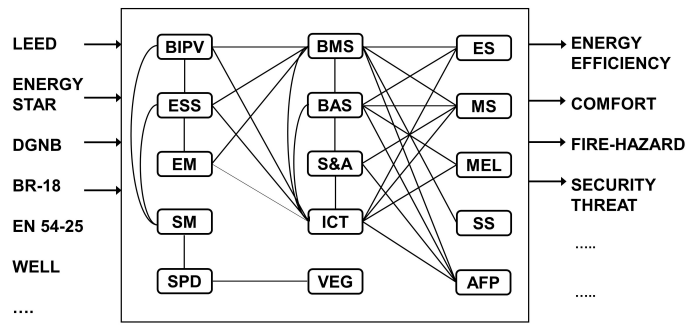

Fig. 1. Contemporary Building Design Paradigm: A Building as System-of-Systems

gyStar (ENERGY, 2011) for systems within the building, occupant's wellness standard such as WELL building construction regulation such as BR-18 C and building fire-safety codes such as EN:54-25 (CEN, 2008) aid in integrated building design.

The aforementioned paradigm in building design emphasize that a careful and systematic approach for functional integration through data/information together with continuous assessment for cooperation of systems in autonomy is paramount to meet the goals. This would otherwise present risks, hazards and threats that would compromise fire-safety, physical security, reliability, interoperability, robustness and integrity of the building as a whole. The statistic d presented in Fig. 2 show that compromise to tire-safety and protection in built-environment particularly in the Nordics arise due to human (e.g. forgetfulness, smoking, cooking, carelessness) or physical (e.g.

\footnotetext{
${ }^{\mathrm{b}} \mathrm{https} / / /$ www.wellcertified.com/

${ }^{\mathrm{c}} \mathrm{https}$ ///bygningsreglementet.dk/

${ }^{\mathrm{d}}$ https://NordicFireStatistics.org/
}

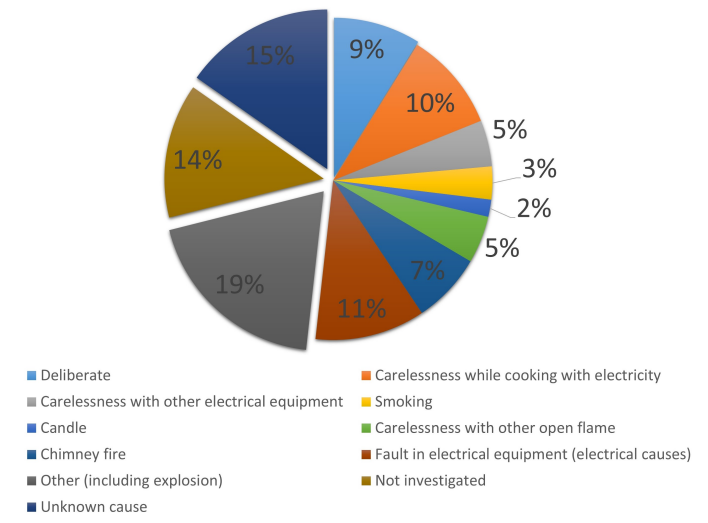

Fig. 2. Building fire distribution in Nordic countries malfunction, miscalibration, miscommissioning, interoperation, ageing) factors. However, this study hypothesize that unprecedented fire-safety hazards in the future buildings can originate from the intersection of physical, social, and cyber spheres and have cross-functional impact. For example, security vs fire-safety, energy-efficiency vs fire-safety, comfort vs fire-safety. The specific aim of this study is to identify such new class of emergent hazards to fire-safety in contemporary building designs using a smart home environment as a case and thus, provide a taxonomy for systematic classification of attack vectors and their impact. This new scientific area is referred as cross-domain fire-safety hazards in this body of work.

\section{Related Work}

A few closely related work are found in literature Heartfield et al. (2018) provide a comprehensive taxonomy for identifying cross-domain hazards in a smart home environment. The taxonomy provides a list of twenty-five cyber-physical attack vectors, together with the illustrations of their potential impact on systems and occupants. However, it is limited to those originating from the cyber sphere. Likewise, Yampolskiy et al. (2013) provide a taxonomy with six dimensions for a structured description of cross-domain attacks on Cyber-Physical Systems (CPS). They consider CPS to have interdependent components in cyber and physical domains, and potential attack surface across their interface.

This study complements previous works by providing a new perspective on a specific case of cross-domain fire-safety hazards in smart buildings, particularly homes. Further, it elucidates how such hazards could surface due to the intersection of all three domains - physical, social and cyber. A scenario-based analysis highlights the knowledge gap in this field of study and furthermore intrigues a discussion on strengthening regulatory codes and standards (e.g. BR18 and EN:5425) to ensure that technological advancements and applications in buildings meet the desired goals in future. In this precinct, the study presents a new taxonomy which aids in the analysis of cross-domain fire-safety attack vectors and their impacts.

\section{Motivation For Taxonomy}

The fire-safety hazards in future buildings can arise due a combination of either cyber, physical, and social means. Thus we have three classes of cross-domain hazards as shown in Fig. 3

(1) Socio-Cyber cross domain

(2) Cyber-Physical cross domain

(3) Socio-Physical cross domain 


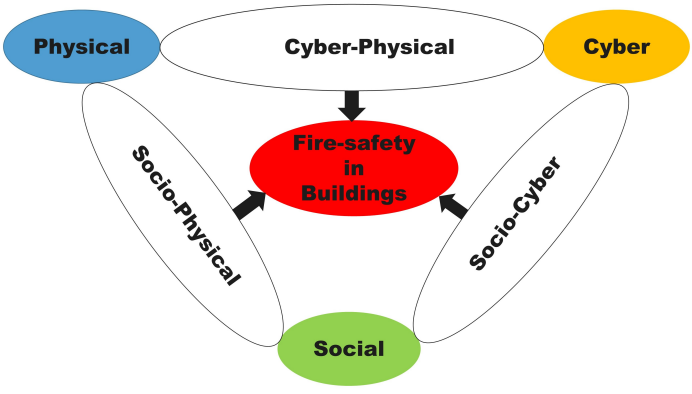

Fig. 3. Cross-domain fire-safety hazards in buildings

A systematic analysis of the aforementioned hazard classes shall require a taxonomy to classify the nature of hazard, means of attack and their impact or consequence. Inspired by Heartfield et al. (2018), this study employs the following set of questions to develop a taxonomy.

- By what means can an adversary compromise fire protection in buildings using smart installations?

- How is fire safety thus compromised?

- What are the consequences?

- How does building occupant's react to compromises in fire-safety?

\section{Case Study: A Typical Smart Home Environment}

With the range of off-the-shelf technologies at disposal, it is relatively easy to build or refurbish a smart building today. Fig. 4 presents an illustrative view of typical smart home with a wide range of capabilities that provides the smartness, as listed below.

- Wireless Home Gateway (e.g. Niko Home Centre, Google Home)

- Work-flow automation apps (e.g. IF-ThisThen-That)

- Voice assistance system (e.g. Google Assistant, Amazon Alexa)

- Wireless door lock

- Wireless smoke detector cum alarm

- Rooftop Photo-voltaic

- Energy Storage System

- CCTV surveillance system (e.g. G4S)

- Smartphone companion app (e.g. Niko Home Control, Google Home, iOS Home)

- Automated Windows and Blinds (e.g.Velux)

- Automated Garage Doors (e.g. Somfy)

- Smart Lighting System (e.g. Philips Hue)

- Pre-programmed 'Smart' modes (e.g. Vacation mode in Niko)

- Smoke Canyons
The home automation has become accessible, in the sense anyone without prior computer programming knowledge can setup automation solutions at ease. Thanks to technological advancements in voice recognition, workflow automation, location-based services etc. This not only benefits smart home enthusiasts but also real-estate companies to offer an integrated smart home with a built-in technology platform. It also enables developing practical application scenarios and package them as solutions to transform passive buildings into smarter homes. Additionally, it allows home owners the ability to modify or reprogram the functionalities of the home automation solution as desired. The aforementioned attributes elucidates that the objectives of integrated smart home packages are convenience, future-proofing, security, safety and energy-efficiency. In Section 5. the authors discuss several cross-domain firesafety hazards that are possible in such a smart home environment.

\section{Discussion: Scenarios and potential fire-safety hazards}

The benefits of having the aforementioned capabilities in a home is clear, but the vulnerabilities could overwhelm the advantages. The stakeholders (building owners and manufacturers) seldom disclose the weaknesses of their offerings e.g. products and services. With standards and regulations still emerging, it is important to develop a taxonomy of threat landscape and their potential consequences related to fire-safety. This section elucidates such vulnerabilities in the integrated design of an illustrative smart home environment depicted in Fig. 4.

\subsection{Integration of ceiling windows and doors with wireless smoke detector through workflow automation app}

The purpose of this integration is to multiplex the role of ceiling windows as smoke ventilation in case of fire through workflow automation app. The integration with smoke detectors shall enable the ceiling windows to automatically open when the fire alarm is triggered. The functional integration is further designed to unlock the door in case of fire, to make sure that residents inside the house can exit. However, the vulnerability of such an integration is the possibility to trigger false fire alarms (by for example remote code execution) which in turn will allow the adversary an easy entry into the house through either the windows or the front door. 


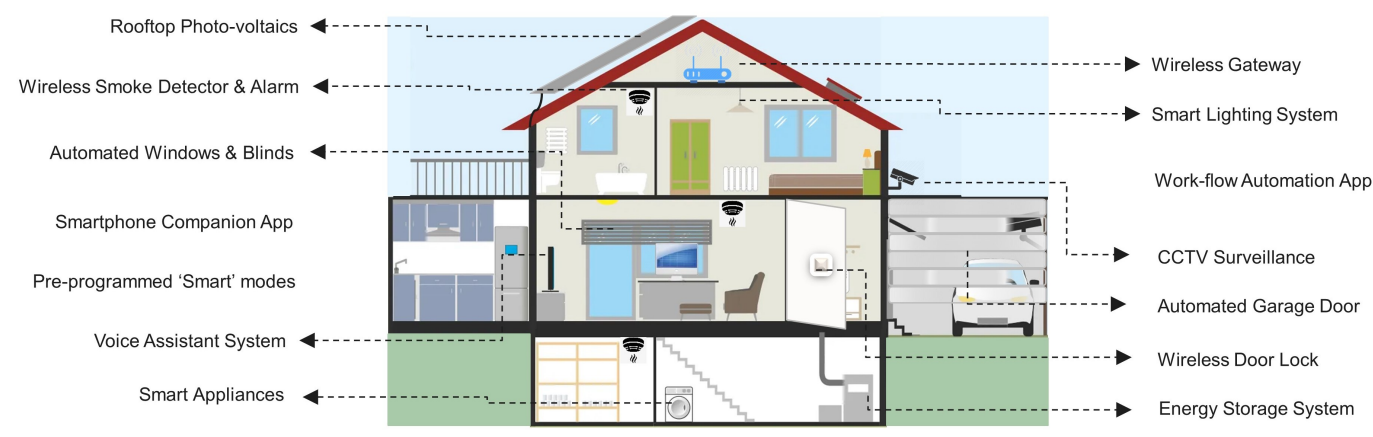

Fig. 4. Typical Smart Home with integrated technologies: An illustration

\subsection{Application of wireless radio-frequency jammer to breach digital security}

The emergence of wireless systems in homes can have an impact on security and fire safety. Systems that are connected via wireless technology are more vulnerable to wireless radio frequency jammers. These jammers can block the wireless communication between different components and by doing so interfere with the data transmission. This can lead to the malfunction of the components and legitimate data not being transferred, in case of e.g. a fire or a burglary. In an extreme situation, an adversary can easily breach security by spoofing smoke alarms in multiple smart homes in a neighborhood, thus redirecting fire service to the compromised neighbourhood and plot a terror attack elsewhere in the city.

\subsection{Integration of wireless door lock and smoke canyons}

The integration of wireless door access control system and smoke canyons is a classical example of technology assisted security system in buildings. In the event of burglary, the smoke canyons are automatically triggered to fog the vision of burglar indoor. In addition, the wireless digital door lock facilitate security professionals to lock-up the burglar (from remote) until the police/security arrives at the scene. This functionality is an example of how integration doubles the security by averting the escape and burglary both. However, the downside of this integration is when the wireless digital door lock either fails to open, thus exacerbate the potential risk (by preventing escape) of lives in the event of a fire.

\subsection{Automation of garage door with smartphone-based location service}

Modern

smart vehicles are equipped with transceivers that are recognized by garage door automation systems. Together they enable opening the door automatically for vehicular entry from a few meters distance. In addition, one could simply automate the workflow (of opening the garage door) using IFTTT ${ }^{\mathrm{s}}$ such that when one drives a car accompanied by a smartphone it recognises the phone and opens the garage door. This definitely adds to the lifestyle convenience. However, this means accessing (stealing) the phone/car gives indirect access to enter the house via garage door. Access to the house is an invitation to an adversary to gain access to sensitive information and/or invaluable property, even to set them on fire.

A related study on workflow automation app examined the security and privacy issues in 19,323 recipes that are publicly available in IFTTT app Surbatovich et al. (2017). It classified risks presented by IFTTT in smart home environment into four categories namely personal, physical, cybersecurity and innocuous. However, the examples presented in this article are potential workflow automation recipes that specifically expose the risk of fire-safety in IFTTT.

\subsection{Integration of energy storage system, wireless door lock and home gateway}

The objective of this functional integration is to ensure that, when energy storage reaches either maximum safe charging or minimum draining limit, it stops charging any further, preventing overheating and fire-hazard. With centralized gateway programmed using workflow automation app to cooperatively control different systems, a

\footnotetext{
${ }^{\mathrm{e}} \mathrm{https}: / /$ ifttt.com
} 
man-in-the-middle attack is just sufficient to introduce a fire hazard. Let us consider a situation where an adversary could introduce a malware that act as a man-in-the middle between rooftop PV and batteries. The attack blinds the home gateway system from the status of actual battery level. This would lead to a situation where batteries either drain or charge beyond permissible limits resulting in over-heat and eventually a spark and fire. The severity of the fire hazard can increase manifold when wireless door lock runs out of built-in power source, locking in the residents and thus preventing timely safe evacuation.

\subsection{Integration of secure and insecure apps}

The centralized integration of physical components (e.g. doors, windows, lights) in homes using digital apps allows the users to have a singlepoint access through a wireless home gateway, for smartness and convenience on one hand. However, on the other hand integrating several wireless components on one network can lead hackers to gain access to all wireless components through a vulnerable component or an insecure app. Such a vulnerable link allows a malware to enter and travel through interconnected systems to eventually infest and disable the Wireless Home Gateway which is the brain of the home automation system. Once the access have been gained the adversary could hack the digital security lock to gain access to home, then blind the cameras (either physically or digitally) and turn-off Wireless Home Gateway, so that communicating any hazard from indoor is compromised.

\subsection{Denial-of-Service of wireless smoke detectors through malware infestation}

The wireless smoke detectors are only useful if their battery is in good health. For safety critical devices, such as smoke detectors, the recommendation is that batteries in wireless smoke detectors last minimum 36 months and indicate end of battery life one month prior (CEN, 2008). However, if an attacker introduces malware into the smart system forcing the wireless smoke detectors to constantly transmit data, the battery would drain in a much faster rate than expected and eventually it would lead to Denial-of-Service (DoS) of smoke detectors. In this case, the malware could force the smoke detectors to trigger a false positive which in turn could overburden remote emergency services or alarming device to which the smoke detectors are connected to.

\subsection{Hacking into energy storage system}

Smart homes equipped with rooftop photovoltaics and energy storage systems are almost self-reliant or sometimes net-energy positive. Such buildings can find good application in smart grids, lowcarbon and smart cities. However, sometimes such buildings can be vulnerable to cross-domain fire-safety hazards. For instance, an adversary can steal (and trade) energy generated and stored in energy storage systems such that it drains batteries below their safety margin, leading to overheating, spark and fire.

\subsection{Integration of smart appliances}

An attacker could reprogram appliances, for example a smart oven or kitchen stove, to produce a high temperature for a long period of time thus creating a risk of ignition which could lead to fire if there are combustibles in the vicinity of the appliance. An adversary could potentially perform this attack by remote code execution, as a Manin-the-Middle attack, or by social engineering.

\subsection{Hacking into wireless gateway}

As mentioned before, centralized control of different subsystems in a smart home makes it unreliable in the event of a cyber-physical attack. For instance, an adversary can hack into the home network and install a malware on the wireless gateway which introduce a Denial-of-Service (DoS) attack. The DoS attack might disable the preprogrammed vacation mode, thus compromising the safety and security of unoccupied home by turning off all appliances and lights.

\section{Taxonomy for cross-domain fire hazards}

This section provides a discussion on the questions asked in section 3 and concludes with table 1 showing an overview of cross-domain fire hazards.

\subsection{By what means can an adversary compromise fire protection in buildings using smart installations?}

An adversary can use jamming devices to disturb radio communication between smart systems in the building. A jamming device could potentially block the radio communication in a much larger area than one building, i.e. an entire neighbourhood. Blocking of radio communication can also happen involuntarily by disturbances from other communication devices in the vicinity, commonly referred as radio-frequency interference.

Another means of attack could be remote code execution (RCE). RCE is the ability to access the computing device and make changes, irrespective of the device's geographical location. Vulnerabilities in a smart installation can provide an adversary with the possibility to execute malicious 
Table 1. Taxonomy of cross-domain fire hazards.

\begin{tabular}{|c|c|c|c|}
\hline Hazard class & Means of attack & Hazard & Fire safety compromise \\
\hline \multirow[t]{4}{*}{ Cyber-physical } & Radio frequency jamming & Loss of communication & Sensor unable to communicate detection of fire \\
\hline & Remote Code Execution & False alarms & Loss of trust in fire protection system \\
\hline & Theft & Denial-of-Service & System safety design not upheld \\
\hline & Man-in-the-Middle & Overheating & Ignition and fire \\
\hline \multirow[t]{3}{*}{ Socio-physical } & Theft & False alarms & Loss of trust in fire protection system \\
\hline & Physical infestation & Overheating & Ignition and fire \\
\hline & & Denial-of-Service & System safety design not upheld \\
\hline \multirow[t]{4}{*}{ Socio-cyber } & Social engineering & Loss of communication & Sensor unable to communicate detection of fire \\
\hline & & False alarms & Loss of trust in fire protection system \\
\hline & & Denial-of-Service & System safety design not upheld \\
\hline & & Overheating & Ignition and fire \\
\hline
\end{tabular}

code into the system and thereby take control of the system with the same privileges as the user running the application. After gaining access to the system by RCE, the attacker will often try to elevate their privileges to increase the control.

An attacker could intercept the communication between two systems in the smart building installation by posing as the original sender, commonly known as a Man-in-the-Middle Attack. The attacker will thus have the original communication and can thereby trick the recipient into thinking that they are still receiving a legitimate message from the sender. (Greenberg, 2015)

Another means of attack is to physically access the smart building system. An attacker could do this by, for example, crawling through void spaces in the walls, lock-picking a door, follow another person into the building (tailgating), posing as contractors or service repairman, or jimmying open improperly installed doors or windows. For a smart home installation the two latter approaches would be most likely. Once the adversary is physically located inside the building, a part of the smart installation could be infested by e.g. a USBstick.

An attacker impersonating e.g. maintenance personnel to access the inside of a building can easily install a malware that can blind the remote agency (e.g. fire service). Social Engineering (SE) have been a well-known phenomenon within sociology for decades. Especially within applied sociology, the term has been described as 'use of scientific methods to suppress the targets volitional capacity' (Murphy, 1981). By using a non- technical approach, a social engineer can 'hack' their targets mind and make them behave in a manner they otherwise would not do. The social engineers simply take advantage of the trusting nature of humans. A Social Engineer is able to bypass the human firewall that is skepticism by using methods that talk to the subconscious. Humans respond well to people who have earned their trust. Persons in uniforms who radiate authority is seldom questioned about their authoritative position in the society. By using this information, SE plays on the encoded trustworthiness of humans and gains access to the real objective. By obtaining trust, the SE gains access and have the ability to make the subject act and do things that they otherwise would not do. For example, by posing as maintenance personnel it would be possible for a non-authorized person to gain access to the Wireless Home Gateway in the house either through the Wireless Home Gateway or via other components that are connected to the Gateway. Thus, buildings with smart installation can get exposed to threats as a result of SE. SE attacks include phishing of confidential information as well as targeted attacks based on gained information. These socially engineered attacks can, for example, be used to interrupt or infect information systems or steal credentials. (Heartfield and Gan. 2016)

Lastly, an adversary could perform a theft operation in order to gain access to the smart installation. Most smart building installations are controlled via a smartphone. If an adversary gets access to this smartphone, access to the smart 
installation is potentially very easy.

\subsection{How is fire safety thus compromised?}

Based on the above mentioned means of attacks to a smart building, the fire safety can be compromised in different ways. First of all, the active fire safety system, which is integrated in the smart building installation, can suffer loss of communication and the fire safety is thereby compromised as it cannot communicate that the sensors detect a fire.

Another possibility is that an adversary can trigger the active fire safety system to communicate false alarms (unnecessary dispatches). This will compromise fire safety by making the system unreliable for the user, i.e. if the fire alarm goes off for random reasons, the credibility and seriousness of the alarm will decrease and the risk of ignoring a real fire incident increases.

The fire safety can also be compromised by a malicious actor rendering one or more devices unavailable by interrupting the device's normal functioning (i.e. DoS attack). Fire safety could be compromised if any of the devices connected to the fire safety system (i.e. smoke detector, alarm system, windows and doors) was targeted with a DoS attack. For example, if the system is designed to open windows in case of fire, to let out smoke, but the automated smart windows are not functioning due to DoS, the result would be that the entire fire safety system is not working according to the intended design.

Lastly, an adversary with control over the smart building installation could trigger appliances to overheat and in worst case cause an ignition leading to explosion and/or fire. Moreover, the consequence could be worse if a failure occurs in a larger battery, on which the smart installation is dependent (Office of Electricity Delivery and Energy Reliability, 2014).

\subsection{What are the consequences?}

Direct consequences of compromised fire safety include, from most severe to moderate: loss of lives, scorch injuries, physical damage to property.

Indirect consequences include damage to health due to smoke inhalation, decrease of psychological well-being, economical loss due to damage to property.

\subsection{How does building occupant's react to fire-safety compromise?}

If compromises becomes common, occupants start reevaluating their trust in the system and it becomes a liability. Numerous false alarms and/or compromises, can lead to great annoyance for the occupants whether it being a cyber-based compromise or other, the occupants will become inured to the alarms and evacuation messages.

\section{Conclusions and Further Work}

Fire has been a known hazard for centuries, but with proliferation of IoT in buildings, the attack surface of fire hazards increased. In this study, we exemplify several unprecedented crossdomain fire hazards that have the potential to compromise safety and security in buildings. Specifically, the taxonomy identified seven means of attack that span across three cross-domains, having the potentially to introduce four different types of fire-safety hazards in future smart buildings.

As the scenarios shows, it is not only the standards within the fire protection systems that has to be revised, but also the standards for entire IoT ecosystem applied to buildings. Up until now, there has been one track for fire protection systems (e.g. EN 54-25), that have had some specific requirements for coverage, interference etc. and building codes (e.g. BR18 in Denmark) that specifies sufficient fire-safety measures viz. safe evacuation, structural load bearing capacity under fire, spread of fire etc. But the hazards could potentially multiply when IoT products overlap/cross over with fire protection systems, as there are no standards for the crossover. The overlap in products, services and their roles become complex when one product have multiple application areas within security and fire (e.g. wireless doors and windows).

Furthermore, buildings in future urban environment will have System-of-Systems making it more difficult to standardize due to complex interconnected systems. The challenge is that no standards covers all aspects - either multiple standards cover one domain or one standard cover multiple domains. This lack of conformity in standards regarding products and domains, could potentially compromise fire safety as developers of technology might use the standard that fits the best, but not necessarily the one that is the safest. Thus, guidelines and standards concerning IoT systems integrated in building designs have to be adapted in synergy with the evolving market for IoT products. The next step is to quantify the cross-functional impact of fire-safety hazards on building's security, energy-efficiency and occupant's comfort. Furthermore, the future work shall aim to develop a framework to assess the risk of integrating IoT-based products for active fireprotection in buildings.

\section{Acknowledgement}

Thanks to Innovation Fund Denmark and Danish Agency for Science and Higher Education for funding this study.

\section{References}

CEN (2008). Fire detection and fire alarm systems - part 25: Components using radio links. 
Council, U. G. B. (2001). Leadership in energy and environmental design (leed).

ENERGY, S. (2011). Energy starß, History: ENERGY STAR.

Greenberg, A. (2015). Hackers remotely kill a jeep on the highway - with me in it. Wired. URL:

https://www.wired.com/2015/07/hackersremotely-kill-jeep-highway/ . Accessed 09-012020.

Heartfield, R. and D. Gan (2016). Social engineering in the internet of everything. Cutter IT Journal 29, 20-29.

Heartfield, R., G. Loukas, S. Budimir, A. Bezemskij, J. R. Fontaine, A. Filippoupolitis, and E. Roesch (2018). A taxonomy of cyberphysical threats and impact in the smart home. Computers \& Security 78, 398-428.

Kennedy, C., N. Ibrahim, and D. Hoornweg (2014). Low-carbon infrastructure strategies for cities. Nature Climate Change 4(5), 343.

Kolokotsa, D. (2016). The role of smart grids in the building sector. Energy and Buildings 116, 703-708.

Kylili, A. and P. A. Fokaides (2015). European smart cities: The role of zero energy buildings. Sustainable Cities and Society 15, 86-95.

Murphy, J. (1981). Applied sociology, social engineering, and human rationality. J. Soc. \& Soc. Welfare 8, 10.

Office of Electricity Delivery and Energy Reliability (2014). Energy storage safety strategic plan.

Silva, B. N., M. Khan, and K. Han (2018). Towards sustainable smart cities: A review of trends, architectures, components, and open challenges in smart cities. Sustainable Cities and Society 38, 697-713.

Surbatovich, M., J. Aljuraidan, L. Bauer, A. Das, and L. Jia (2017). Some recipes can do more than spoil your appetite: Analyzing the security and privacy risks of iftt recipes. In Proceedings of the 26th International Conference on World Wide Web, pp. 1501-1510.

Wang, N., P. E. Phelan, C. Harris, J. Langevin, B. Nelson, and K. Sawyer (2018). Past visions, current trends, and future context: A review of building energy, carbon, and sustainability. Renewable and Sustainable Energy Reviews 82, 976-993.

Yampolskiy, M., P. Horvath, X. D. Koutsoukos, Y. Xue, and J. Sztipanovits (2013). Taxonomy for description of cross-domain attacks on cps. In Proceedings of the 2nd ACM international conference on High confidence networked systems, pp. 135-142. ACM. 